\title{
Conventional and Functional MR Imaging of Peripheral Nerve Sheath Tumors: Initial Experience
}

\author{
S. Demehri, A. Belzberg, J. Blakeley, and L.M. Fayad
}

\begin{abstract}
BACKGROUND AND PURPOSE: Differentiating benign from malignant peripheral nerve sheath tumors can be very challenging using conventional MR imaging. Our aim was to test the hypothesis that conventional and functional MR imaging can accurately diagnose malignancy in patients with indeterminate peripheral nerve sheath tumors.
\end{abstract}

\begin{abstract}
MATERIALS AND METHODS: This institutional review board-approved, Health Insurance Portability and Accountability Act-compliant study retrospectively reviewed 61 consecutive patients with 80 indeterminate peripheral nerve sheath tumors. Of these, 31 histologically proved peripheral nerve sheath tumors imaged with conventional (unenhanced T1, fluid-sensitive, contrast-enhanced T1-weighted sequences) and functional MR imaging (DWI/apparent diffusion coefficient mapping, dynamic contrast-enhanced MR imaging) were included. Two observers independently assessed anatomic (size, morphology, signal) and functional (ADC values, early arterial enhancement by dynamic contrast-enhanced MR) features to determine interobserver agreement. The accuracy of MR imaging for differentiating malignant from benign was also determined by receiver operating characteristic analysis.
\end{abstract}

RESULTS: Of 31 peripheral nerve sheath tumors, there were 9 malignant (9\%) and 22 benign ones (81\%). With anatomic sequences, average tumor diameter ( $6.3 \pm 1.8$ versus $3.9 \pm 2.3 \mathrm{~mm}, P=.009)$, ill-defined/infiltrative margins (77\% versus $32 \% ; P=.04)$, and the presence of peritumoral edema $(66 \%$ versus $23 \%, P=.01)$ were different for malignant peripheral nerve sheath tumors and benign peripheral nerve sheath tumors. With functional sequences, minimum $\mathrm{ADC}\left(0.47 \pm 0.32 \times 10^{-3} \mathrm{~mm}^{2} / \mathrm{s}\right.$ versus $\left.1.08 \pm 0.26 \times 10^{-3} \mathrm{~mm}^{2} / \mathrm{s} ; P<.0001\right)$ and the presence of early arterial enhancement $(50 \%$ versus $11 \% ; P=.03)$ were different for malignant peripheral nerve sheath tumors and benign peripheral nerve sheath tumors. The minimum ADC (area under receiver operating characteristic curve was $0.89 ; 95 \%$ confidence interval, 0.73-0.97) and the average tumor diameter (area under the curve $=0.8 ; 95 \% \mathrm{Cl}, 0.66-0.94$ ) were accurate in differentiating malignant peripheral nerve sheath tumors from benign peripheral nerve sheath tumors. With threshold values for minimum $A D C \leq 1.0 \times 10^{-3} \mathrm{~mm}^{2} / \mathrm{s}$ and an average diameter of $\geq 4.2 \mathrm{~cm}$, malignancy could be diagnosed with $100 \%$ sensitivity $(95 \%$ $\mathrm{Cl}, 66.4 \%-100 \%)$.

CONCLUSIONS: Average tumor diameter and minimum ADC values are potentially important parameters that may be used to distinguish malignant peripheral nerve sheath tumors from benign peripheral nerve sheath tumors.

ABBREVIATIONS: AUC $=$ area under the curve; BPNST $=$ benign peripheral nerve sheath tumor; DCE $=$ dynamic contrast-enhanced; MPNST $=$ malignant peripheral nerve sheath tumor; NF-1 = neurofibromatosis type 1; PNST = peripheral nerve sheath tumor; ROC = receiver operating characteristic analysis

M alignant peripheral nerve sheath tumors (MPNSTs) have a much smaller prevalence compared with benign peripheral nerve sheath tumors (BPNSTs) in the general population, ${ }^{1}$ though a higher prevalence of MPNSTs occurs in the setting of neurofibromatosis type $1(\mathrm{NF}-1),{ }^{2}$ where patients have a $10 \%$ lifetime risk of developing MPNST. ${ }^{3}$ Distinguishing BPNSTs

Received October 17, 2013; accepted after revision December 16.

From The Russell H. Morgan Department of Radiology and Radiological Science (S.D., L.M.F.) and Department of Neurosurgery (A.B.), Johns Hopkins University School of Medicine, Baltimore, Maryland; and The Johns Hopkins Hospital Comprehensive Neurofibromatosis Center (J.B.), Department of Neurology, The Johns Hopkins Hospital, Baltimore, Maryland. from MPNSTs is important, because most sporadic tumors are BPNSTs, and unnecessary biopsies of benign tumors can be obviated if the accurate characterization of peripheral nerve sheath tumors (PNSTs) by noninvasive imaging techniques is available. Differentiating BPNSTs from MPNSTs can be very challenging because not only do their clinical features overlap but character-
Please address correspondence to Shadpour Demehri, MD, Johns Hopkins University School of Medicine, Musculoskeletal Radiology, Russell H. Morgan Department of Radiology and Radiological Science, 601 N. Caroline St, JHOC 5165, Baltimore, MD 21287; e-mail: demehri2001@yahoo.com

三 Indicates article with supplemental on-line table.

http://dx.doi.org/10.3174/ajnr.A3910 
istics by conventional MR imaging sequences are also shared. ${ }^{4-6}$ In patients with NF-1, benign neurofibromas with small $<5-\mathrm{cm}^{7}$ well-defined margins, a target sign, homogeneous signal intensity, and the absence of necrosis are considered as benign "determinate" tumors ${ }^{8}$; but without such characteristics, with large or increasing size of a lesion with time or significant uptake by positron-emission tomography, PNSTs in patients with NF-1 are defined as "indeterminate" and potentially malignant. ${ }^{9-11}$ Additionally, sporadic PNSTs are also deemed "indeterminate", ${ }^{12}$ especially if they are large, contain heterogeneous signal or internal necrosis, or exhibit ill-defined or infiltrative margins. ${ }^{7}$ Patients with indeterminate PNSTs, which may be benign or malignant, are typically referred for an image-guided percutaneous biopsy or surgical removal for definitive histologic diagnosis, while patients with determinate BPNSTs do not necessarily require a biopsy and may be referred for follow-up. ${ }^{8,12}$

The addition of functional MR imaging sequences with DWI and dynamic contrast-enhanced (DCE) MR imaging to conventional MR imaging has been suggested as a useful approach to the assessment of soft-tissue tumors. ${ }^{13}$ In particular, quantitative DWI with apparent diffusion coefficient mapping has been investigated for the characterization of musculoskeletal lesions, by demonstrating differences in cellularity between benign and malignant tumors. ${ }^{14}$ With DCE-MR imaging, specific patterns of enhancement have been associated with benign and malignant soft-tissue lesions. ${ }^{15,16}$ Hence, the purpose of this study was to test the hypothesis that conventional and functional MR imaging can accurately diagnose malignancy in patients with indeterminate peripheral nerve sheath tumors.

\section{MATERIALS AND METHODS Subject Population}

This retrospective study was approved by the institutional review board, and informed consent was waived. A study population of consecutive soft-tissue tumors with features suggestive of "indeterminate" PNSTs was created by reviewing consecutive MR imaging studies obtained in our institution or outside MR imaging reviewed in our multidisciplinary nerve tumor clinics from May 2008 and January 2013. "Determinate" PNSTs were excluded (PNSTs in patients with NF-1 of a small size of $<5 \mathrm{~cm}$, a target sign, homogeneous signal, and absence of peritumoral edema or necrosis). Of the remaining 80 "indeterminate" PNSTs, we excluded the following: Sixteen (20\%) tumors had no available functional imaging; $18(22.5 \%)$ had no available contrast-enhanced imaging $(n=12)$ and/or DWI/ADC mapping $(n=8)$. In 3 patients (3.8\%), the DWI sequences were nondiagnostic due to susceptibility or motion artifacts; and 12 presumed benign PNSTs had no histologic proof. The remaining 31 PNSTs were included in the study.

\section{MR Imaging Acquisition Protocols}

Conventional MR Imaging. MR imaging was performed at $3 \mathrm{~T}$ (Siemens, Erlangen, Germany) by using a flexible phased array body-matrix coil and included the following sequences: T1weighted (TR/TE, 790/15 ms; section thickness, $5 \mathrm{~mm}$ ), fat-suppressed T2-weighted (TR/TE, 3600/70 ms; section thickness, 5 $\mathrm{mm}$ ), and short tau inversion recovery (TR/TE, 3600/70 ms; sec- tion thickness, $5 \mathrm{~mm}$; axial plane), followed by unenhanced and gadolinium-based contrast agent-enhanced 3D fat-suppressed T1-weighted imaging (volumetric interpolated breath-hold examination, isotropic resolution; TR/TE, 4.6/1.4 ms; flip angle, $9.5^{\circ}$; section thickness, $1 \mathrm{~mm}$; coronal plane with axial and sagittal reconstructions; $0.1-\mathrm{mmol} / \mathrm{kg}$ gadolinium-based contrast agent; Siemens Medical Solutions, Malvern, Pennsylvania).

Functional MR Imaging. Diffusion weighted-imaging with ADC mapping (TR/TE, 760/80 ms; section thickness, $5 \mathrm{~mm}$; b-values $=50,400$, and $800 \mathrm{ss} / \mathrm{mm}^{2}$ ) of the entire tumor was performed in the axial plane in all cases. Dynamic contrast-enhanced MR imaging was also performed in 26 of 31 tumors, immediately before and after the administration of the gadolinium-based contrast agent (time-resolved angiography with interleaved stochastic trajectories; TR/TE 2.5/0.9 ms; injection rate, $2-5 \mathrm{~mL} / \mathrm{s}$; flip angle, $20^{\circ}$; FOV , $45 \times 45 \mathrm{~cm}$; acquisition plane, usually coronal; temporal resolution, 10 seconds for total of 5 minutes).

\section{Image Interpretation}

Two independent observers interpreted MR imaging features independently to determine interobserver agreement. Discrepancies were subsequently resolved by consensus to determine the accuracy of specific MR imaging features in differentiating benign and malignant PNSTS. During interpretation, the observers were blinded and had no knowledge of the patients' clinical information, the histologic results of the PNSTs, and the follow-up status of each tumor.

\section{Conventional MR Imaging}

The following lesion features were categorized by anatomic sequences: Maximum tumor diameter measurements were performed in 3 planes (craniocaudal, anteroposterior, transverse), and an "average diameter" of each tumor was determined. Next, signal intensity (hypointense, isointense, or hyperintense to muscle) and heterogeneity $(<25 \%, 25 \%-50 \%,>50 \%)$ on $\mathrm{T} 1-$ and T2-weighted sequences were assessed. The shape (round/ovoid, irregular) and margin type (well-defined; partially defined, $>50 \%$ of lesion margin defined; ill-defined, $<50 \%$ of its margin defined; infiltrative) were identified. The presence or absence of the split fat sign, target sign, peritumoral edema, encasement or invasion into the adjacent vessels, periosteal/cortical involvement, bone marrow involvement, and joint extension were recorded. The presence or absence of enhancement following contrast administration on static postcontrast images and the percentage of tumor enhancement $(<25 \%, 25 \%-50 \%, 50 \%-75 \%$, and $>75 \%)$ were detailed.

\section{Functional MR Imaging}

Using DWI/ADC mapping, each observer placed the largest oval or round region of interest entirely within each tumor on 3 axial sections, at the cranial, caudal, and central portions of the tumor. The minimum and mean ADC values for each axial section were reported, and the minimum and average mean ADC values for the 3 sections were determined. Using DCE-MR imaging, we recorded the presence or absence of early arterial enhancement. 


\section{Statistical Analysis}

A Pearson correlation $t$ test and Spearman rank order correlation test were performed to evaluate interobsever agreement for continuous and categoric data, respectively. To compare continuous and categoric MR imaging measurements for features of BPNSTs and MPNSTs, we used a 2-tailed Student $t$ test and Fisher exact test, respectively. Receiver operating characteristic analysis (ROC) was performed to determine the accuracy of each measurement by using consensus measurements.

Using the ROC curve, we obtained optimal threshold values for continuous measurements, as the maximum Youden index, defined as threshold value $=$ sensitivity $-(1$-specificity $)$ of each measurement. ${ }^{17}$ Statistical analysis of the data was performed with a statistical software program MedCalc for Windows (MedCalc Software, Mariakerke, Belgium). A $P$ value $\leq .05$ was considered significant.

Table 1: Demographic characteristics of patients with biopsyproved benign and malignant nerve sheath tumors ${ }^{\mathrm{a}}$

\begin{tabular}{lcc}
\hline & MPNST $(\boldsymbol{n}=9)$ & BPNST $(\boldsymbol{n}=\mathbf{2 2})$ \\
\hline No. of patients & 8 & $21^{\mathrm{a}}$ \\
Sex & & \\
$\quad$ Male & 4 & 13 \\
$\quad$ Female & 4 & 8 \\
Age (median) (range) (yr) & $38,18-54$ & $52,13-78$ \\
NF-1 & 9 & 4 \\
Benign pathologies & - & Schwannoma $(n=14)$ \\
& & Neurofibroma $(n=6)$ \\
& & Perineuroma $(n=1)$ \\
& & Ganglioneuroma $(n=1)$ \\
\hline
\end{tabular}

Note:- indicates not applicable

a One patient with NF-1 had 2 MPNSTs and 1 patient with NF-1 had 1 MPNST and 2 BPNSTs.

Table 2: Comparison between benign and malignant peripheral nerve sheath tumors using conventional and functional MRI and determination of interobserver agreement

\begin{tabular}{|c|c|c|c|c|}
\hline & $\begin{array}{c}\text { MPNST } \\
(n=9)\end{array}$ & $\begin{array}{l}\text { BPNST } \\
(n=22)\end{array}$ & $P$ Value & $\begin{array}{c}\text { Interobserver } \\
\text { Agreement }^{\mathrm{a}}\end{array}$ \\
\hline \multicolumn{5}{|l|}{ Diameter $(\mathrm{cm})$} \\
\hline Maximum (mean) & $8 \pm 2.4$ & $5.6 \pm 3.8$ & .17 & 0.89 \\
\hline Average (mean) & $6.3 \pm 1.8$ & $3.9 \pm 2.3$ & .009 & 0.82 \\
\hline \multicolumn{5}{|l|}{ Conventional MRI } \\
\hline $\mathrm{Tl}$ (Hyponintense to muscle) & $0 \%(0 / 9)$ & $14 \%(3 / 22)$ & .22 & 0.90 \\
\hline T2 (Hyperintense to the muscle) & $100 \%(9 / 9)$ & $100 \%(21 / 22)$ & .99 & 0.98 \\
\hline \multicolumn{5}{|l|}{ Following features present: } \\
\hline Ill-defined or infiltrative margins & $77 \%(7 / 9)$ & $32 \%(7 / 22)$ & .04 & 0.66 \\
\hline Irregular shape & $0 \%(0 / 9)$ & $12.5 \%(3 / 22)$ & .528 & 0.80 \\
\hline Split fat sign & $10 \%(1 / 9)$ & $33 \%(8 / 22)$ & .11 & 0.21 \\
\hline Target sign & $10 \%(1 / 9)$ & $21 \%(5 / 22)$ & .51 & 0.92 \\
\hline $\mathrm{Tl}$ heterogeneity $(<50 \%)$ & $0 \%(0 / 9)$ & $4 \%(1 / 22)$ & .49 & 0.76 \\
\hline T2 heterogeneity $(<50 \%)$ & $55 \%(5 / 9)$ & $29 \%(7 / 22)$ & .31 & 0.71 \\
\hline Peritumoral edema & $66 \%(6 / 9)$ & $23 \%(5 / 22)$ & .01 & 0.71 \\
\hline Eccentric to the nerve & $55 \%(5 / 9)$ & $42 \%(10 / 22)$ & .18 & 0.53 \\
\hline Vascular encasement & $11 \%(1 / 9)$ & $0 \%(0 / 22)$ & .65 & 0.95 \\
\hline Periosteal/cortical invasion & $11 \%(1 / 9)$ & $0 \%(0 / 22)$ & .65 & 1 \\
\hline Bone marrow invasion & $0 \%(0 / 9)$ & $0 \%(0 / 22)$ & .99 & 1 \\
\hline Joint extension & $0 \%(0 / 9)$ & $0 \%(0 / 22)$ & .99 & 1 \\
\hline Delayed contrast enhancement & $100 \%(9 / 9)$ & $92 \%(22 / 22)$ & .39 & 0.70 \\
\hline \multicolumn{5}{|l|}{ Functional MRI } \\
\hline \multicolumn{5}{|l|}{ ADC values $\left(\times 10^{-3} \mathrm{~mm}^{2} / \mathrm{s}\right)$} \\
\hline Average (mean) & $1.57 \pm 0.27$ & $1.7 \pm 0.28$ & .22 & 0.90 \\
\hline Minimum (mean) & $0.47 \pm 0.32$ & $1.08 \pm 0.26$ & $<.0001$ & 0.89 \\
\hline \multicolumn{5}{|l|}{ DCE-MRI } \\
\hline Early arterial enhancement & $50 \%(4 / 8)$ & $11 \%(2 / 18)$ & .03 & 0.81 \\
\hline
\end{tabular}

a Spearman rank-order correlation coefficient. .0001) (Table 1).

\section{RESULTS}

\section{Subject Characteristics}

Of 31 biopsy-proved PNSTs, 22 were benign and 9 were MPNSTs. The patients with MPNSTs (mean age, 38 years; range, 18-54 years) were younger than patients with BPNSTs (mean age, 52 years of age; range, $13-78$ years $)(P=.02)$. All MPNSTs $(9 / 9)$ and only $22 \%$ of BPNSTs $(5 / 22)$ occurred in patients with NF-1 $(P=$

\section{MR Imaging Features}

There was moderate-to-high interobserver agreement for MR imaging measurements and determinations by using both anatomic and functional sequences (Table 2). Among the measurements obtained by using conventional MR imaging (Table $2)$, the average tumor diameter $(6.3 \pm 1.8$ versus $3.9 \pm 2.3 \mathrm{~cm}$, $P=.009)$, the presence of peritumoral edema $(6 / 9,66 \%$ versus $5 / 22,23 \% ; P=.01)$, and the presence of ill-defined/infiltrative margins $(7 / 9,77 \%$ versus $7 / 22,32 \% ; P=.04)$ were significantly different in MPNSTs than in BPNSTs, respectively.

With functional MR imaging sequences, the minimum ADC values were significantly lower in MPNSTs than in BPNSTs $\left(0.47 \pm 0.32 \times 10^{-3} \mathrm{~mm}^{2} / \mathrm{s}\right.$ versus $1.08 \pm 0.26 \times 10^{-3} \mathrm{~mm}^{2} / \mathrm{s}$, respectively; $P<.0001$ ), while average $\mathrm{ADC}$ values were not significantly different. With DCE-MR imaging, 50\% of MPNSTs (4/8, DCE-MR was not performed for 1 MPNST) demonstrated early arterial enhancement compared with only $11 \%$ of BPNSTs $(n=2 / 18, P=.03)$.

\section{Diagnostic Performance with ROC}

With conventional MR imaging, the average tumor diameter showed the highest accuracy for differentiating MPNSTs and BPNSTs (area under the curve; AUC $=0.83$; 95\% CI, 0.660.94). Other important diagnostic features included maximum lesion diameter, peritumoral edema, and tumor margin, which offered AUCs of 0.77 (95\% CI, $0.59-0.90), 0.68$ (95\% CI, 0.49-0.84), and $0.68(95 \% \mathrm{CI}, 0.49-0.84)$, respectively (On-line Table).

With functional MR imaging, the minimum ADC value (AUC $=0.89 ; 95 \%$ CI, $0.73-0.97, P=.02$ ) was a more accurate parameter in differentiating BPNSTs and MPNSTs than the average ADC value $(\mathrm{AUC}=0.63 ; 95 \% \mathrm{CI}, 0.43-0.76)($ Fig 1$)$. The AUC for the presence of early arterial enhancement by using DCE-MR was 0.69 (95\% CI, 0.46-0.86).

With the Youden index, the threshold values for the features with the highest diagnostic performance (minimum ADC of $1.0 \times 10^{-3} \mathrm{~mm}^{2} / \mathrm{s}$ and an average diameter of $4.2 \mathrm{~cm}$ ) were combined to stratify (Fig. 2) the "indeterminate" PNSTs, and ROC was further used to determine the accuracy of this combination of an- 
atomic and functional MR imaging data. For PNSTs with both minimum $\mathrm{ADC}$ value $\geq 1.0 \times 10^{-3} \mathrm{~mm}^{2} / \mathrm{s}$ and an average diameter of $<4.2 \mathrm{~cm}$, the sensitivity and negative predictive value for diagnosing malignancy were $100 \%$ (95\% CI, 66\%-100\%) and 100\%, respec-

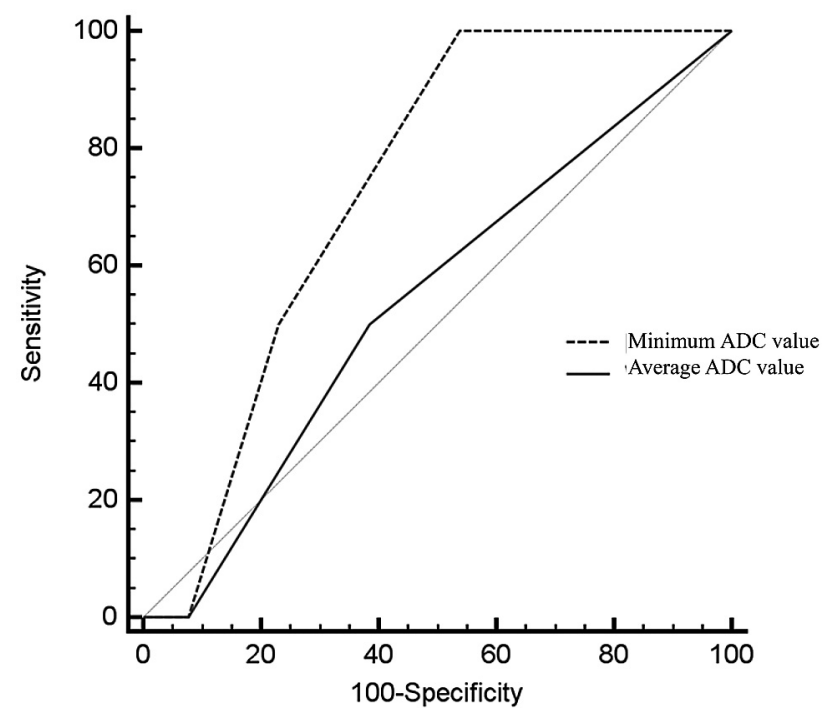

FIG 1. ROC for $A D C$ values by using DWI in differentiation of BPNSTs and MPNSTs. The minimum ADC value (AUC $=0.89 ; 95 \%$ $\mathrm{Cl}, 0.73-0.97 ; P=.02$ ) was a more accurate parameter in differentiating BPNSTs and MPNSTs than the average ADC value (AUC = $0.63 ; 95 \% \mathrm{Cl}, 0.43-0.76$ ). (The ADC values plotted here are consensus values determined from a mean of the measurements made by each reader.) tively. Conversely, for PNSTs with both minimum ADC values $<1.0 \times 10^{-3} \mathrm{~mm}^{2} / \mathrm{s}$ and average diameter of $\geq 4.2 \mathrm{~cm}$, the specificity and positive predictive value for malignancy were $77 \%$ (95\% CI, 54\%-92\%) and 64\%, respectively (Figs 3 and 4).

\section{DISCUSSION}

Any improvement in the diagnostic accuracy of noninvasive imaging for characterizing the population of "indeterminate" PNSTs is valuable, given the high prevalence of benign PNSTs $(22 / 31,71 \%)$ that may be unnecessarily referred for biopsy rather than follow-up. In this study, we showed that the combined anatomic and functional MR imaging features of "indeterminate" PNSTs may be helpful to rule out malignancy.

With anatomic MR imaging features, results of the current study are in agreement with prior investigations, in that a large tumor diameter is a useful feature for differentiating BPNSTs and MPNSTs. Our study also showed that within "indeterminate" PNSTs, the average diameter $(P=.009)$ and not the maximum diameter $(P=.17)$ is significantly larger in MPNSTs in comparison with BPNSTs. This result may be due to the fact that typical fusiform BPNSTs usually grow along the nerve and, therefore, may have a large maximum diameter along the nerve of origin, but average measurements that incorporate length in the other 2 planes may be smaller than the maximum diameter alone. Using ROC, in our study, we observed a threshold value of $4.2 \mathrm{~cm}$, a value that is close to the commonly used metric of $5 \mathrm{~cm}$ for differentiating benign and malignant soft-tissue tumors. $^{7,18}$

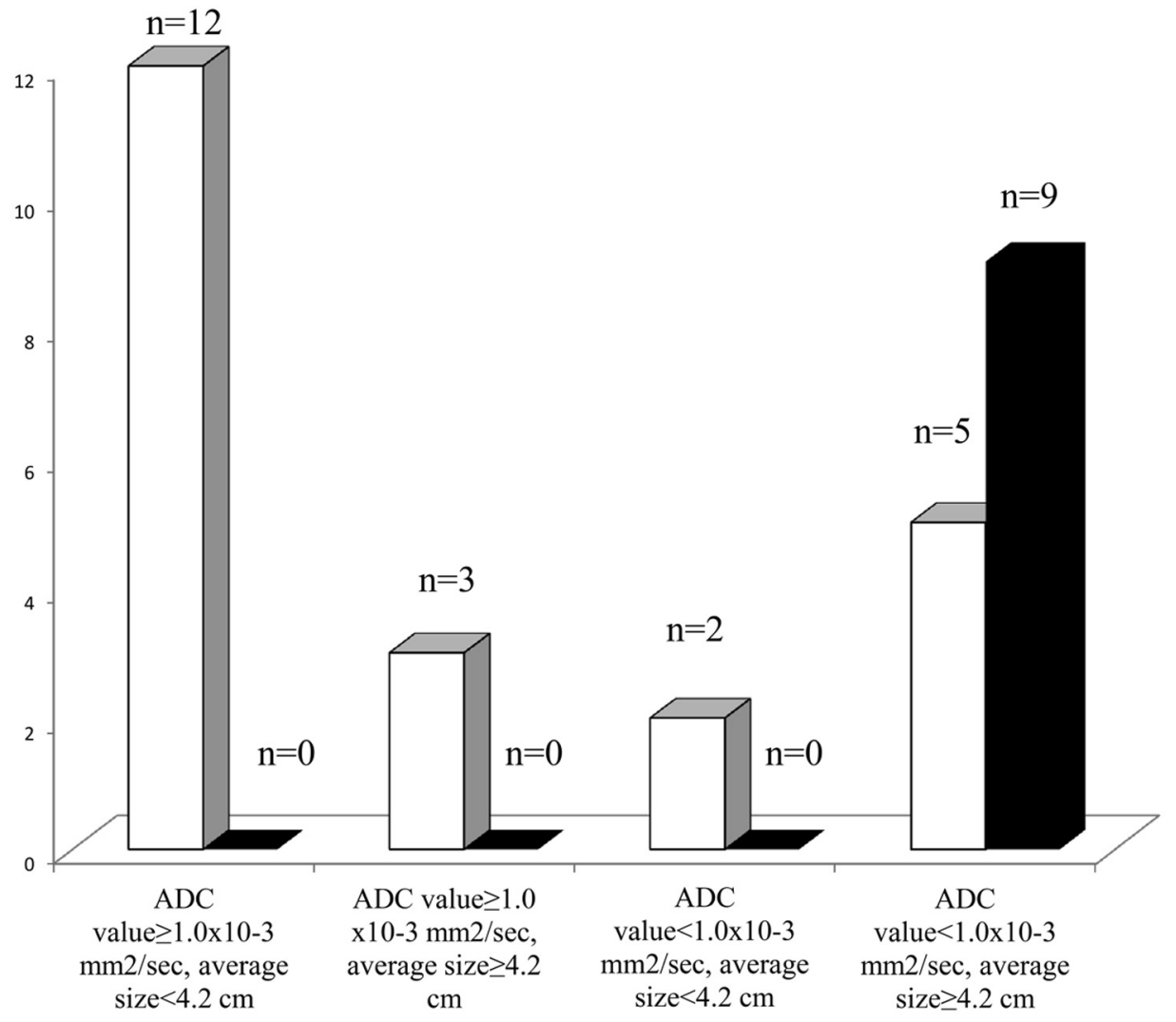

$\square$ BNST

- PNST

FIG 2. Distribution bar plots for BPNSTs and MPNSTs stratified on the basis of threshold values obtained from ROC of anatomic (average diameter $=4.2 \mathrm{~cm}$ ) and functional (minimum $A D C=1.0 \times 10^{-3} \mathrm{~mm}^{2} / \mathrm{s}$ ) MR imaging sequences. 

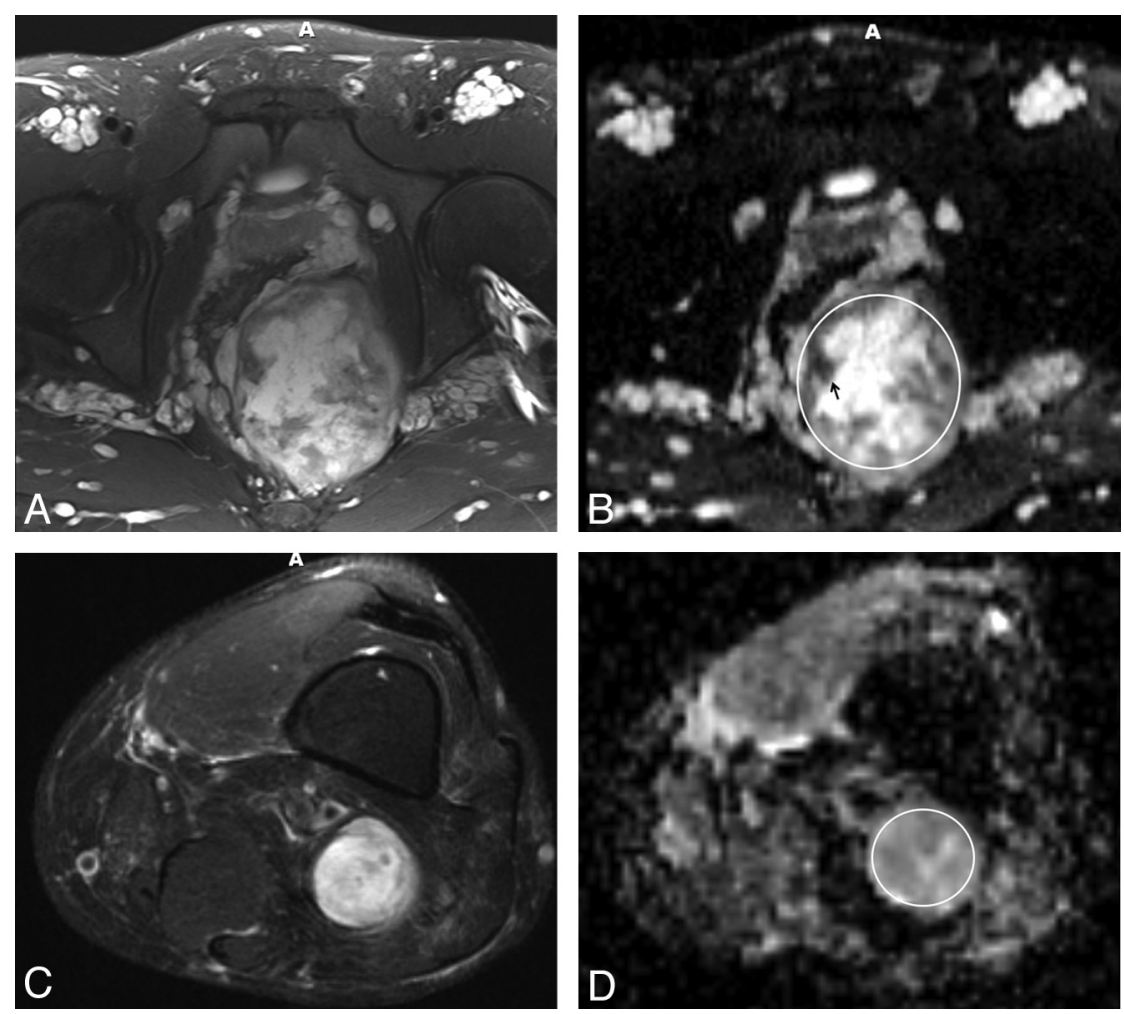

FIG 3. A 21-year-old man with a history of NF-1 and an "indeterminate" PNST. Axial T2weighted image $(A)$ and ADC map $(B)$ both show heterogeneous signal with a large pelvic mass with an average diameter of $8.7 \mathrm{~cm}$ and a minimum ADC value of $0.4 \times 10^{-3} \mathrm{~mm}^{2} / \mathrm{s}$ within this mass (arrow), subsequently diagnosed as MPNST. For comparison, a benign neurofibroma in 54-year-old man with a history of NF-1 is shown in the distal thigh, with an average diameter of $3.4 \mathrm{~cm}(C)$ and a minimum $A D C$ value of $1.0 \times 10^{-3} \mathrm{~mm}^{2} / \mathrm{s}(D)$. The circle indicates the region of interest for ADC measurements.
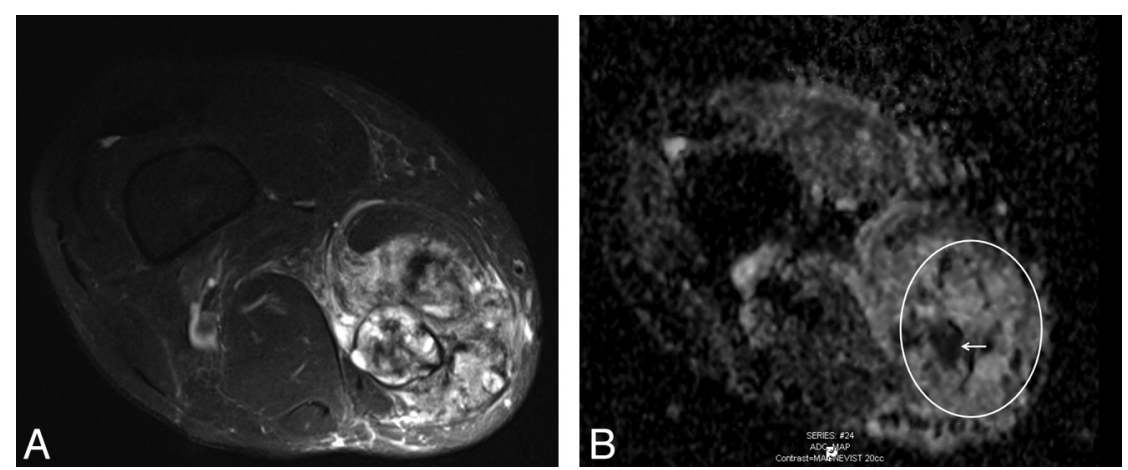

FIG 4. "Indeterminate" PNST in the distal right thigh of a 65-year-old man with an average size of $9.0 \mathrm{~cm}$ shown on an axial T2-weighted image $(A)$ and a minimum ADC value of $0.1 \times$ $10^{-3} \mathrm{~mm}^{2} / \mathrm{s}\left(B\right.$, arrow). Although PNSTs with a minimum ADC value $\leq 1.0 \times 10^{-3} \mathrm{~mm}^{2} / \mathrm{s}$ and/or an average diameter of $>4.2 \mathrm{~cm}$ can still be benign, these tumors must proceed to histologic diagnosis to rule out malignancy. In this case, a benign schwannoma was found. The circle indicates the region of interest for ADC measurements.

Other anatomic features that have been explored in the literature include the presence of peritumoral edema and heterogeneous enhancement following contrast administration, both of which were shown to be supporting features for MPNST in our study. However, signal heterogeneity on noncontrast sequences and cystic changes on contrast-enhanced sequences have also been described as important differentiating features between neurofibromas and MPNSTs, but our study did not support this observation. A large number of schwannomas (14/22, 63\%) were included in our study, and such tumors are more likely to be "indeterminate," because they can be very heterogeneous and contain cystic regions (so-called ancient schwannomas), unlike the more uniform signal characteristics in typical neurofibromas. ${ }^{19}$ Similarly, there was no significant difference in the presence of a target sign in MPNSTs (10\%) and BPNSTs (21\%), though the lack of a target sign has been reported as a useful diagnostic sign of MPNST in a population comparing neurofibromas and MPNSTs. ${ }^{4}$

Because the histologic evaluation of PNSTs commonly shows increased cellularity in MPNSTs, it follows that DWI is helpful in differentiating BPNSTs and MPNSTs. In particular, the results of our study show that the minimum ADC value is a better predictor of malignancy than the average ADC value in PNSTs, in sporadic cases and in patients with NF-1. This observation can be explained by the heterogeneity in cellularity present within both benign and malignant PNSTs, which can result in a large range of intralesional ADC values, making the minimum value more valuable than the diluted average $\mathrm{ADC}$ value. In addition, in patients with NF-1, MPNSTs typically arise within neurofibromas $^{2}$ and are associated with the foci of highest cellularity. Minimum ADC values may also be helpful for imageguided biopsy planning because targeting areas with the lowest ADC values can improve the diagnostic yield. ${ }^{20}$ In our study, a threshold minimum ADC value of $1.0 \times$ $10^{-3} \mathrm{~mm}^{2} / \mathrm{s}$ was determined as an accurate quantitative measure of malignancy, as has been suggested anecdotally in softtissue tumors in general. ${ }^{14}$ Similar to MPNSTs, BPNSTs with high cellularity, such as schwannomas, ${ }^{19}$ can also demonstrate low minimum ADC values, as found in 4 schwannomas and 1 ganglioneuroma with minimum ADC values $<1.0 \times 10^{-3} \mathrm{~mm}^{2} / \mathrm{s}$.

Another functional technique explored in this study was DCE-MR imaging. Although nonspecific, ${ }^{19}$ malignant tumors more often demonstrate early arterial enhancement than benign tumors. ${ }^{13}$ In our study, early arterial enhancement was detected in MPNSTs (50\%) more commonly than in BPNSTs (11\%, $P=.03)$, suggesting that the presence of early arterial enhancement may also be a useful sign for distinguishing MPNSTs from BPNSTs, when present.

To combine the information obtained from conventional and functional MR imaging, by using ROC and the Youden index, we 
stratified PNSTs on the basis of the threshold values for average lesion diameters $(\geq 4.2 \mathrm{~cm})$ and minimum ADC values $\left(\leq 1.0 \times 10^{-3}\right.$ $\mathrm{mm}^{2} / \mathrm{s}$ ). With these thresholds, for PNSTs with an average diameter $<4.2 \mathrm{~cm}$ and a minimum $\mathrm{ADC}$ value of $\geq 1.0 \times 10^{-3} \mathrm{~mm}^{2} / \mathrm{s}$, the possibility of an MPNST is unlikely. Hence, the impact of these results on clinical care, while yet to be definitively determined, may be anticipated. In our retrospective study, $38 \%$ of the indeterminate tumors met these threshold criteria; therefore, conservative management and continued surveillance of these tumors may have been sufficient (because these tumors were histologically proved benign). However, these imaging features are by no means diagnostic for malignancy, and in our cohort, $36 \%$ of "indeterminate" tumors in this subgroup were diagnosed as BPNSTs (Fig 4). There was also high interobserver agreement for minimum ADC values $(r=0.89)$ and average-diameter $(r=0.82)$ measurements.

This study had limitations. First, our retrospective study had a nonrandomized design; therefore, the presence of selection bias was unavoidable. Second, in our study, MPNSTs composed 29\% (9/31) of the "indeterminate" PNSTs, a much higher incidence than in the general population. This is likely due to our inclusion criteria, because MPNSTs are more likely than BPNSTs to undergo a histologic evaluation. Third, a limited analysis of DCE-MR imaging data was performed, because we only investigated the presence of early arterial enhancement for distinguishing MPNSTs from BPNSTs; the latter technique, though not quantitative, has recently been shown to be adequate for assessing soft-tissue sarcomas. ${ }^{21}$ Further quantitative analysis of tumor perfusion can also be performed, which may enhance the differentiation of benign and malignant tumors, but it is a cumbersome analysis that is not as easily performed clinically as the quantification of ADC maps. Finally, 1 observer (Laura M. Fayad) was directly involved in the diagnosis and management of some tumors included in this study. Therefore, it is possible that this observer remembered at least some of the cases during interpretation. However, we determined the accuracy of MR imaging features in the differentiation of BPNSTs and MPNSTs by using a consensus interpretation of 2 observers.

\section{CONCLUSIONS}

This study provides initial evidence that the addition of functional imaging with DWI and ADC mapping to a conventional MR imaging examination has a potential role for distinguishing benign and malignant PNSTs and may impact the clinical care of patients with these common tumors.

Disclosures: Allen Belzberg—UNRELATED: Grants/Grants Pending: Children's Tumor Foundation, ${ }^{*}$ Comments: Principal Investigator on International Schwannomatosis Database. Jaishri Blakeley — UNRELATED: Travel/Accommodations/Meeting Expenses Unrelated to Activities Listed: BiPar (2007), Novartis (2008), Comments: travel expenses to professional meetings (American Academy of Neurology, American Society of Clinical Oncology) for educational presentations. Laura M. Fayad — UNRELATED: Grants/Grants Pending: AUR GE Radiology Research Academic Fellowship (2010), ${ }^{*}$ Siemens (2011), ${ }^{*}$ Comments: for MR spectroscopy work. *Money paid to the institution.

\section{REFERENCES}

1. Kransdorf MJ. Benign soft-tissue tumors in a large referral population: distribution of specific diagnoses by age, sex, and location. AJR Am J Roentgenol 1995;164:395-402

2. Ducatman BS, Scheithauer BW, Piepgras DG, et al. Malignant peripheral nerve sheath tumors: a clinicopathologic study of $\mathbf{1 2 0}$ cases. Cancer 1986;57:2006-21
3. Evans DG, Baser ME, McGaughran J, et al. Malignant peripheral nerve sheath tumors in neurofibromatosis 1. J Med Genet 2002;39: 311-14

4. Bhargava R, Parham DM, Lasater OE, et al. MR imaging differentiation of benign and malignant peripheral nerve sheath tumors: use of the target sign. Pediatr Radiol 1997;27:124-29

5. Kehrer-Sawatzki H, Kluwe L, Fünsterer C, et al. Extensively high load of internal tumors determined by whole body MRI scanning in a patient with neurofibromatosis type 1 and a non-LCR-mediated 2-Mb deletion in 17q11.2. Hum Genet 2005;116:466-75

6. Koşucu P, Ahmetoĝlu A, Cobanoĝlu U, et al. Mesenteric involvement in neurofibromatosis type 1: CT and MRI findings in two cases. Abdom Imaging 2003;28:822-26

7. Wasa J, Nishida Y, Tsukushi S, et al. MRI features in the differentiation of malignant peripheral nerve sheath tumors and neurofibromas. AJR Am J Roentgenol 2010;194:1568-74

8. Frassica FJ, Khanna JA, McCarthy EF. The role of MR imaging in soft tissue tumor evaluation: perspective of the orthopedic oncologist and musculoskeletal pathologist. Magn Reson Imaging Clin N Am 2000;8:915-27

9. Beert E, Brems H, Daniëls B, et al. Atypical neurofibromas in neurofibromatosis type $\mathbf{1}$ are premalignant tumors. Genes Chromosomes Cancer 2011;50:1021-32

10. Friedrich RE, Gawad K, Derlin T, et al. Surgery for atypical plexiform neurofibromas of the trunk in NF1 with high standardised uptake value (SUV) in positron-emission tomography (PET) expressing podoplanin: a long-term follow-up. Anticancer Res 2012;32:4547-51

11. Meany H, Dombi E, Reynolds J, et al. 18-fluorodeoxyglucose-positron emission tomography (FDG-PET) evaluation of nodular lesions in patients with neurofibromatosis type 1 and plexiform neurofibromas $(\mathrm{PN})$ or malignant peripheral nerve sheath tumors (MPNST). Pediatr Blood Cancer 2013;60:59-64

12. Papp DF, Khanna AJ, McCarthy EF, et al. Magnetic resonance imaging of soft-tissue tumors: determinate and indeterminate lesions. J Bone Joint Surg Am 2007;89(suppl 3):103-15

13. Fayad LM, Jacobs MA, Wang X, et al. Musculoskeletal tumors: how to use anatomic, functional, and metabolic MR techniques. Radiology 2012;265:340-56

14. Maeda M, Matsumine A, Kato H, et al. Soft-tissue tumors evaluated by line-scan diffusion-weighted imaging: influence of myxoid matrix on the apparent diffusion coefficient. J Magn Reson Imaging 2007;25:1199-204

15. Van Rijswijk CS, Geirnaerdt MJ, Hogendoorn PC, et al. Soft-tissue tumors: value of static and dynamic gadopentetate dimeglumineenhanced MR imaging in prediction of malignancy. Radiology 2004;233:493-502

16. Hawighorst H, Libicher M, Knopp MV, et al. Evaluation of angiogenesis and perfusion of bone marrow lesions: role of semiquantitative and quantitative dynamic MRI. J Magn Reson Imaging 1999; 10:286-94

17. Perkins NJ, Schisterman EF. The inconsistency of "optimal" cutpoints obtained using two criteria based on the receiver operating characteristic curve. Am J Epidemiol 2006;163:670-75

18. Rydholm A, Berg N. Size, site and clinical incidence of lipoma: factors in the differential diagnosis of lipoma and sarcoma. Acta Orthop Scand 1983;54:929-34

19. Ahlawat S, Blakeley J, Montgomery E, et al. Schwannoma in neurofibromatosis type 1: a pitfall for detecting malignancy by metabolic imaging. Skeletal Radiol 2013;42:1317-22

20. Rosenkrantz AB, Kim S, Lim RP, et al. Prostate cancer localization using multiparametric MR imaging: comparison of Prostate Imaging Reporting and Data System (PI-RADS) and Likert scales. Radiology 2013;269:482-92

21. Fayad LM, Mugera C, Soldatos T, et al. Technical innovation in dynamic contrast-enhanced magnetic resonance imaging of musculoskeletal tumors: an MR angiographic sequence using a sparse kspace sampling strategy. Skeletal Radiol 2013;42:993-1000 